\title{
Estudo preliminar de características oceanográficas e meteorológicas presentes na pesca da tainha no sul do Brasil
}

\author{
Argeu Vanz \\ Rafael Censi Borges** \\ Maikon Passos Amilton Alves \\ Fábio Ziemann Lopes* \\ Clóvis Roberto Levien Correa
}

\section{Resumo}

Este trabalho tem como objetivo analisar preliminarmente as características oceanográficas e meteorológicas (TSM, ondas, vento e ciclones) presentes na pesca da tainha no período de 2001-2010. Os resultados mostram que as TSM frias favorecem a pesca, mas volumes significativos podem ser capturados em anos com TSM altas. As ondas com alturas maiores de 1,5m são comuns no período da pesca. A predominância de vento do quadrante sul não necessariamente determina maior volume de tainha pescado. A frequência de ciclones maior em maio não interfere fortemente na pesca. Os volumes pescados revelam nos últimos anos uma alternância de safras boas e ruins. A carência de dados deixou lacunas que precisam ser preenchidas em trabalhos futuros; esses devem envolver outras variáveis e escalas temporais e espaciais diferentes das adotadas no presente estudo.

Palavras-chave: Tainha; Pesca; Oceanografia; Meteorologia.

\footnotetext{
Mestre em Oceanografia Física, Química e Biológica. Oceanólogo na Epagri/Ciram (argeuvanz@epagri.sc.gov.br).

** Gestor Ambiental e Técnico em Meteorologia. Técnico em Meteorologia na Epagri/Ciram (censi@epagri.sc.gov.br).

*** Geógrafo e Técnico em Meteorologia. Técnico em Meteorologia na Epagri/Ciram (maikonalves@epagri.sc.gov.br).

******** Mestre em Meteorologia (fzlopes1@gmail.com).

Mestre em Meteorologia. Meteorologista na Epagri Ciram (clovis@epagri.sc.gov.br)
}

Geosul, Florianópolis, v. 27, n. 54, p 55-76, jul./dez. 2012 
VANZ, A. et al. Estudo preliminar de características oceanográficas e...

Preliminary study of oceanographic and meteorological features

present in mullet fishing in southern Brazil

\section{Abstract}

The present study aims to preliminary analyze the oceanographic and meteorological characteristics (SST, waves, wind and cyclones) in the mullet fishing during the period from $2001-2010$. The results show that the cold SST favor fishing, but significant amounts can be captured in years with high SST. Waves with heights greater than $1.5 \mathrm{~m}$ are common in the fishery. The predominance of wind from the south quadrant does not necessarily determine the greatest amount of mullet fish. The greater frequency of cyclones in May does not interfere heavily on fishing. Volumes caught in recent years reveal an alternation of good and bad harvests. The lack of data left gaps that need to be filled in future studies, these should involve other variables, and temporal and spatial scales different from those adopted in this study.

Key words: Mullet; Fisheries; Oceanography; Meteorology.

\section{Introdução}

As tainhas são peixes que pertencem à Familia Mugilidae e são comumente encontrados em estuários e lagoas costeiras. Alimentam-se de matéria vegetal retirada do substrato onde vivem. Possuem ampla capacidade de regulação osmótica o que permite adaptarem-se às variações de salinidade (VIEIRA, 1985; MENEZES \& FIGUEIREDO, 1985).

Mugil platanus é a espécie mais capturada no sul do Brasil e pode atingir $1 \mathrm{~m}$ de comprimento e $6 \mathrm{~kg}$ de biomassa (IBAMA/ICMBIO/CEPSUL 2007). Os juvenis dessa espécie ficam no ambiente estuarino durante todo o ano e são mais abundantes nas áreas de criação da Lagoa dos Patos durante o inverno e primavera (VIEIRA 1985). No outono os adultos abandonam o 
VANZ, A. et al. Estudo preliminar de características oceanográficas e...

estuário, estimulados pela queda na temperatura da água e aumento da salinidade e iniciam a sua migração reprodutiva. A desova ocorre em águas quentes $\left(19^{\circ}-21^{\circ} \mathrm{C}\right)$ afastadas da costa, em latitude próxima de $27^{\circ} \mathrm{S}$, entre o final do outono e no inverno (VIEIRA 1985; VIEIRA \& SCALABRIN 1991). Realizada a desova, os ovos e as larvas são transportados pelas correntes para a zona de arrebentação migrando ao longo da costa para o estuário da Lagoa dos Patos (VIEIRA 1991).

A captura das tainhas envolve aspectos biológicos da espécie, humanos, meteorológicos e oceanográficos. No caso da pesca artesanal o olheiro é responsável pela identificação do cardume. Ao avistar o cardume ele dá o sinal e começa a corrida para cercar o cardume que é arrastado para a praia por dezenas de pessoas. Remadores experientes e a agilidade em soltar a rede são igualmente importantes. Fatores ambientais como o vento do quadrante sul, mar com ondas baixas (menos de $1,0 \mathrm{~m}$ ) e águas oceânicas frias são características ideais para a captura. $O$ vento sul faz com que a água fria do extremo Sul do Brasil chegue mais rápida a costa catarinense e com ela, os cardumes de tainhas. $\mathrm{O}$ mar calmo e as ondas menores de $1,0 \mathrm{~m}$ possibilitam que os pescadores ultrapassem a zona de arrebentação e fechem o cerco.

$\mathrm{Na}$ pesca industrial as condições ambientais não são tão determinantes quanto na artesanal, pois os barcos possuem potentes motores e vários instrumentos/equipamentos que facilitam a procura e a captura das tainhas.

Este trabalho tem como objetivo analisar preliminarmente características oceanográficas e meteorológicas presentes no período de captura da tainha nos estados de Santa Catarina (SC) e Rio Grande do Sul (RS) entre anos 2001-2010.

\section{Material e Métodos}

Os dados meteorológicos utilizados nesse trabalho foram obtidos do banco de dados da Epagri/Ciram. Os dados diários da Temperatura da Superfície do Mar (TSM) foram obtidos através de 
VANZ, A. et al. Estudo preliminar de características oceanográficas e...

satélite sensor Advanced Very High Resolution Radiometer (AVHRR) canal infravermelho, in situ a partir de navios e bóias e, inclui um ajuste de grande escala dos desvios de satélite com relação ao de dados in situ. Os dados são interpolados pelo método Interpolação Ótima (OI). As informações de TSM possuem uma grade espacial de $0.25^{\circ}$ e resolução temporal de 1 dia. As análises de TSM estão disponíveis em arquivos individuais diários no endereço: ftp://eclipse.ncdc.noaa.gov/pub/OI-dailyv2/NetCDF/YYYY/AVHRR, onde YYYY é o ano correspondente.

O volume de tainha capturado para o Estado de SC foi obtido do relatório para o ordenamento da pesca da tainha na região sudeste/sul do Brasil (IBAMA/ICMBIO/CEPSUL 2007; IBAMA 2007).

Foram utilizados a direção e velocidade do vento em $(\mathrm{m} / \mathrm{s})$ de dez estações meteorológicas automáticas sendo 5 do RS e 5 de SC (TABELA 1). A direção e a velocidade do vento das estações meteorológicas foram medidas por um anemômetro a 10 metros de altura do solo. Os dados da direção e velocidade registrados para cada hora foram à média dos valores registrados a cada 15 segundos, totalizando 240 observações em cada hora.

Foram calculadas as médias diárias da velocidade do vento para os dias com pelo menos 20 horários de observação sendo os demais dias descartados. Os meses analisados foram aquele que coincidem com a pesca da tainha no estado, maio, junho e junho de cada ano e a partir dos dados diários foram calculadas as médias da velocidade de cada mês. Com este mesmo critério também foi calculada a média da velocidade dos quadrantes.

A direção predominante do vento diária foi calculada também para os dias com pelo menos 20 horários de observação. Sendo a predominante a que obteve o maior número de casos. Os meses utilizados na direção predominante e na porcentagem das direções dos quadrantes foram: maio, junho e julho. 
VANZ, A. et al. Estudo preliminar de características oceanográficas e...

TABELA 1: Estação, localização, altitude, fonte e período inicial e final da série histórica das estações meteorológicas automáticas utilizadas no estudo.

\begin{tabular}{|c|c|c|c|c|c|}
\hline Estação & ocal. & Alt. (m) & Fonte & Início & Fim \\
\hline Itajaí-SC & $\begin{array}{l}26^{\circ} 54^{\prime} 00^{\prime \prime} \\
48^{\circ} 39^{\prime} 00^{\prime \prime}\end{array}$ & 2 & (1) & $14 / 3 / 2001$ & $31 / 7 / 2010$ \\
\hline Chuí-RS & $\begin{array}{l}33^{\circ} 44^{\prime} 30^{\prime \prime} \\
53^{\circ} 22^{\prime} 18^{\prime \prime}\end{array}$ & 10 & (2) & $16 / 7 / 2005$ & $15 / 3 / 2008$ \\
\hline Most.-RS & $\begin{array}{l}31^{\circ} 14^{\prime} 52^{\prime \prime} \\
50^{\circ} 54^{\prime} 24^{\prime \prime}\end{array}$ & 2 & (2) & $13 / 7 / 2005$ & $28 / 8 / 2005$ \\
\hline S. F. Sul-SC & $\begin{array}{l}26^{\circ} 13^{\prime} 58^{\prime \prime} \\
48^{\circ} 31^{\prime} 55^{\prime \prime}\end{array}$ & 12 & (3) & $22 / 12 / 2003$ & $31 / 7 / 2010$ \\
\hline Laguna-SC & $\begin{array}{l}28^{\circ} 36^{\prime} 13^{\prime \prime} \\
48^{\circ} 48^{\prime} 46^{\prime \prime}\end{array}$ & 17 & (4) & $1 / 6 / 2008$ & $31 / 7 / 2010$ \\
\hline S. José-SC & $\begin{array}{l}27^{\circ} 36^{\prime} 07^{\prime \prime} \\
48^{\circ} 37^{\prime} 11^{\prime \prime}\end{array}$ & 1,84 & (4) & $2 / 8 / 2005$ & $31 / 7 / 2010$ \\
\hline Itapoá-SC & $\begin{array}{l}26^{\circ} 04^{\prime} 52^{\prime \prime} \\
48^{\circ} 38^{\prime} 30^{\prime \prime}\end{array}$ & 2 & (4) & $18 / 6 / 2007$ & $31 / 7 / 2010$ \\
\hline Torres-RS & $\begin{array}{l}29^{\circ} 21^{\prime} 00^{\prime \prime} \\
49^{\circ} 44^{\prime} 00^{\prime \prime}\end{array}$ & 4,66 & (4) & $31 / 5 / 2006$ & $31 / 7 / 2010$ \\
\hline Rio Grande-RS & $\begin{array}{l}32^{\circ} 02^{\prime} 00^{\prime \prime} \\
52^{\circ} 06^{\prime} 00^{\prime \prime}\end{array}$ & 2,46 & (4) & 2/8/2005 & $31 / 7 / 2010$ \\
\hline Tramandaí RS & $\begin{array}{l}30^{\circ} 00^{\prime} 97^{\prime \prime} \\
50^{\circ} 13^{\prime} 53^{\prime \prime}\end{array}$ & 1 & (4) & $1 / 5 / 2008$ & $31 / 7 / 2010$ \\
\hline
\end{tabular}

(1) Epagri/Milênio/Cepsul/Ibama (2) Epagri/Milênio/CHM (3) Epagri/Milênio

(4) INMET

Para analisar a TSM foi aplicado ANOVA para verificar se as médias da TSM dos anos, para cada ponto, eram diferentes, e posteriormente, foi utilizado o teste de Duncan para comparação das médias duas a duas. Foi escolhido esse teste devido ao seu poder efetivo para detectar diferenças quando essas existem. $\mathrm{O}$ teste de Duncan apresenta como característica estatística uma probabilidade baixa de rejeitar a hipótese de que as médias são iguais, quando são iguais, e uma probabilidade alta de rejeitar essa mesma hipótese, quando é falsa. 
VANZ, A. et al. Estudo preliminar de características oceanográficas e...

\section{Resultados e discussão}

\section{Temperatura da superfície do mar - TSM}

A FIGURA 1 mostra localização dos pontos onde foram obtidas as TSM. Ao total, foram 9 pontos analisados dos quais, 4 no RS (P1,P2,P3,P4), um no limite entre RS e SC (P5), 3 em SC (P6, P7, P8) e o último no limite entre SC e Paraná (PR) (P9).

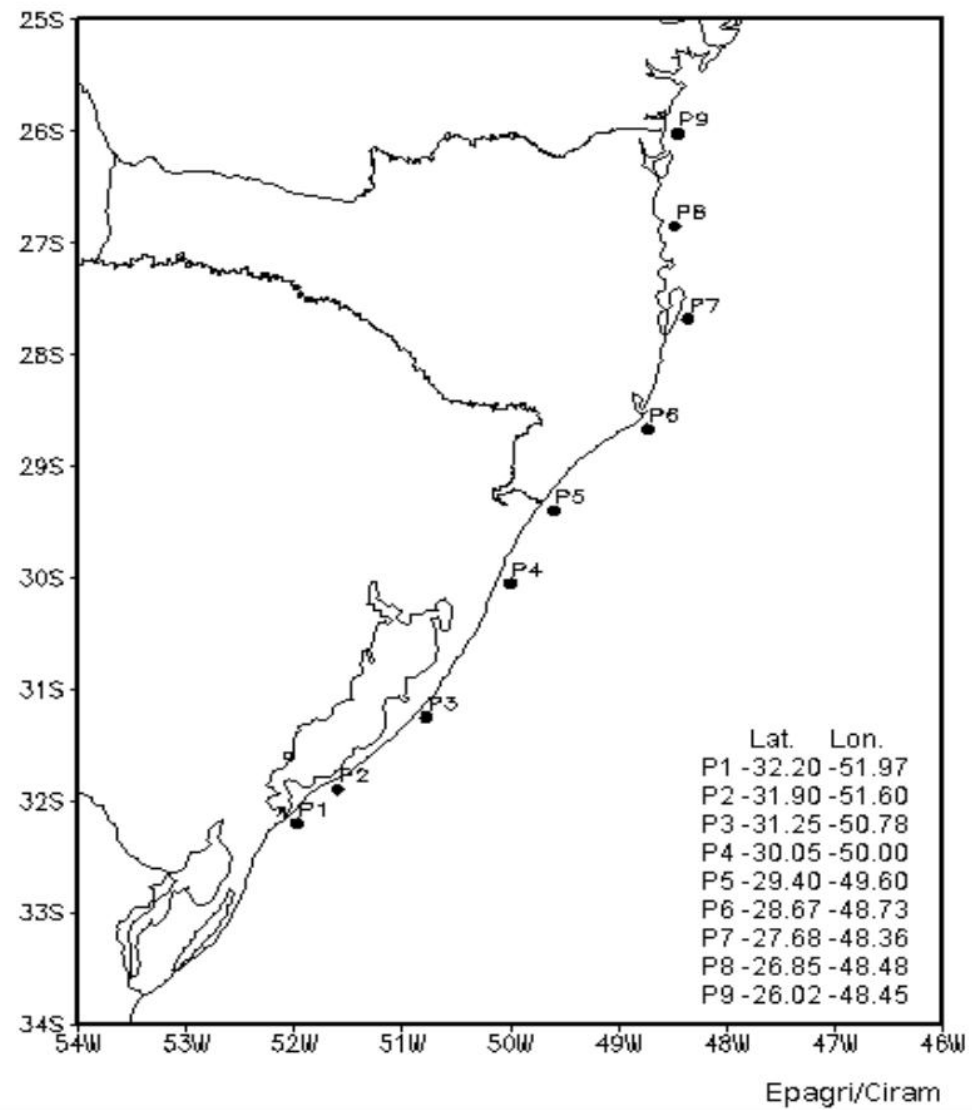

FIGURA 1: Localização dos pontos da temperatura da superfície do mar (TSM) 
VANZ, A. et al. Estudo preliminar de características oceanográficas e...

O comportamento da média da TSM no período da pesca da tainha (maio, junho e julho) nos anos de estudo em cada ponto está representado na FIGURA 2. A TSM tem como tendência aumentar em direção as baixas latitudes.
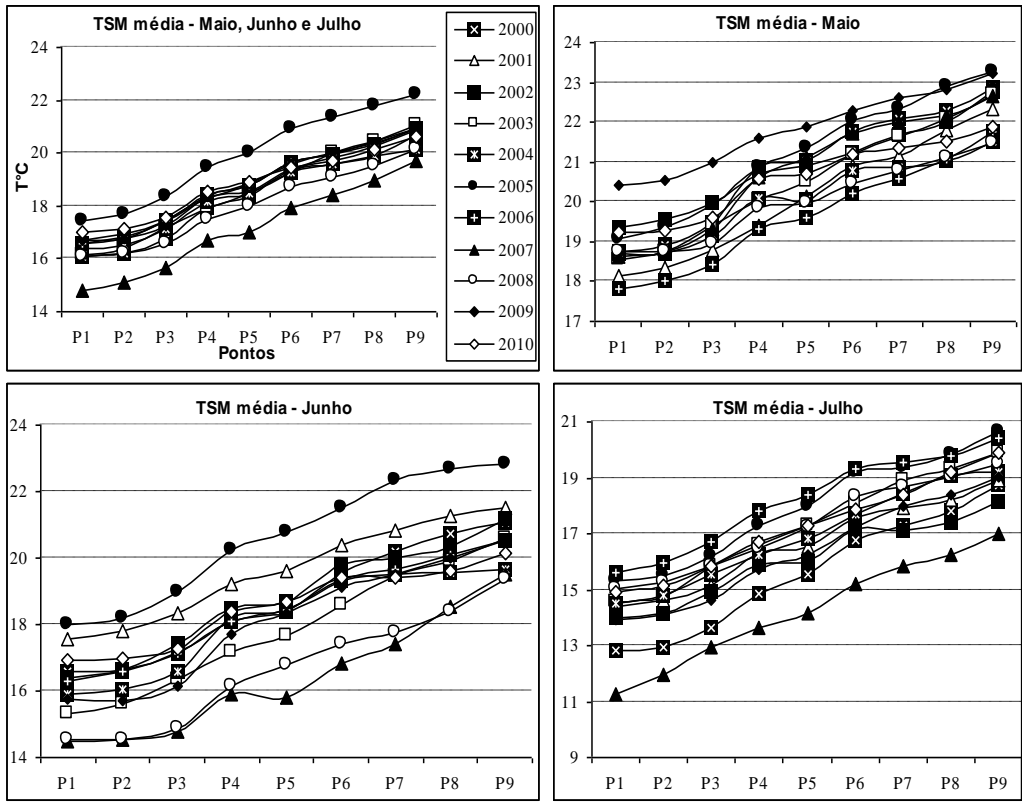

FIGURA 2: Valores de TSM médios para o período dos 3meses, maio, junho e julho.

A Figura 2 mostra que o ano de 2005 foi o ano mais quente e 2007, o mais frio. Os demais anos apresentam TSM mais próximas ás de 2005 (quentes) no P1 e se aproximam da TSM de 2007 (frias) no P9. Os meses que mais contribuíram para que o ano de 2005 tivesse TSM altas foram junho e julho atingindo valores acima de $22^{\circ} \mathrm{C}$ no P9. Em junho desse ano, a TSM tende a ser maior na costa catarinense, P6, P7, P8, P9. Em junho, o ano de 2001 também apresentou TSM alta comparativamente aos demais anos. 
VANZ, A. et al. Estudo preliminar de características oceanográficas e...

$\mathrm{O}$ ano de 2007 destaca-se por apresentar TSM baixas em todos os pontos. A média mensal mostra que ocorre uma diminuição acentuada da TSM de maio a junho e permanecendo baixa em julho. O ano de 2008 apresentou comportamento semelhante ao de 2007, porém com TSM um pouco mais altas. Analisando o comportamento mensal de 2008, verifica-se que a temperatura diminuiu acentuadamente de maio a junho, porém em julho ela permaneceu igual a junho interrompendo sua trajetória de queda, diferentemente do que ocorreu com 2007 que continuou esfriando nesse mês. Entretanto, estatisticamente não há diferença significativa entre esses anos como descrito a seguir.

A análise estatística da TSM mensal mostra que junho foi o mês que mais contribuí para que anos de 2005, 2007 e 2008 apresentassem um comportamento distinto dos demais. Em maio, 2009 forma um grupo isolado dos demais nos primeiros cinco pontos (P1, P2, P3, P4, P5). Outros grupos, compostos por um maior numero de anos também foram registrados (TABELA 2). Estatisticamente, 2007 e 2008 forma um grupo que se distingue dos demais em todos os pontos, exceto para o P9, quando 2004 se junta a eles. Os anos de 2005 e 2001 separam-se dos demais e em alguns pontos formam grupos independentes entre si (TABELA 2).

Além desses 4 anos, outros grupos apareceram em determinados pontos, composto por apenas um ano ou, em outros casos, formados por vários anos. Julho é marcado por um grupo composto por 2007 (frio) e em outro, formado por 2005 e 2006 com característica de TSM (quente) comparativamente aos demais anos. Ainda nesse mês, o ano de 2000 forma um grupo isolado com TSM fria nos primeiros pontos (P1, P2, P3, P4). 
VANZ, A. et al. Estudo preliminar de características oceanográficas e...

TABELA 2: Estatística da comparação das médias pelo teste Duncan. Onde:

P1...P9 - são os pontos;

Em cada ponto, anos com letras iguais pertencem ao mesmo grupo;

Número entre os parênteses representa a quantidade de grupos formados - são mostrados na tabela só os grupos que se separam completamente dos demais.

\begin{tabular}{|c|c|c|c|c|c|c|c|c|c|}
\hline $\begin{array}{l}\text { Ponto } \\
\text { 3mes. }\end{array}$ & $\begin{array}{c}\text { P1 } \\
(5) \\
2007^{\mathrm{a}} \\
2005^{\mathrm{b}}\end{array}$ & $\begin{array}{c}\mathrm{P} 2 \\
(5) \\
2007^{\mathrm{a}} \\
2005^{\mathrm{b}}\end{array}$ & $\begin{array}{c}\mathrm{P} 3 \\
(6) \\
2007^{\mathrm{a}} \\
2005^{\mathrm{b}}\end{array}$ & $\begin{array}{c}\mathrm{P} 4 \\
(5) \\
2007^{\mathrm{a}} \\
2008^{\mathrm{c}} \\
2005^{\mathrm{b}}\end{array}$ & $\begin{array}{c}\text { P5 } \\
(5) \\
2007^{\mathrm{a}} \\
2005^{\mathrm{b}}\end{array}$ & $\begin{array}{c}\text { P6 } \\
(4) \\
2007^{\mathrm{a}} \\
2008^{\mathrm{c}} \\
\text { outros }^{\mathrm{a}} \text { anos }^{\mathrm{d}} \\
2005^{\mathrm{b}}\end{array}$ & $\begin{array}{c}\text { P7 } \\
(5) \\
2007^{\mathrm{a}} \\
2008^{\mathrm{c}} \\
2005^{\mathrm{b}}\end{array}$ & $\begin{array}{c}\mathrm{P} 8 \\
(5) \\
2007^{\mathrm{a}} \\
2008^{\mathrm{c}} \\
2005^{\mathrm{b}}\end{array}$ & $\begin{array}{c}\mathrm{P} 9 \\
(5) \\
2007^{\mathrm{a}} \\
2004^{\mathrm{c}} \\
2008^{\mathrm{c}} \\
2005^{\mathrm{b}}\end{array}$ \\
\hline Maio & $\begin{array}{c}(6) \\
2009^{\mathrm{a}}\end{array}$ & $\begin{array}{c}(6) \\
2009^{\mathrm{a}}\end{array}$ & $\begin{array}{c}(6) \\
2009^{\mathrm{a}}\end{array}$ & $\begin{array}{c}(4) \\
2010^{\mathrm{b}} \\
2007^{\mathrm{b}} \\
2002^{\mathrm{b}} \\
2000^{\mathrm{b}} \\
2005^{\mathrm{b}} \\
2009^{\mathrm{a}}\end{array}$ & $\begin{array}{c}(7) \\
2009^{\mathrm{a}}\end{array}$ & $\begin{array}{c}\text { (5) } \\
-\end{array}$ & $\begin{array}{c}\text { (8) } \\
-\end{array}$ & $\begin{array}{c}(5) \\
2004^{\mathrm{c}} \\
2006^{\mathrm{c}} \\
2008^{\mathrm{c}} \\
2009^{\mathrm{a}} \\
2005^{\mathrm{a}}\end{array}$ & $\begin{array}{c}(5) \\
2004^{\mathrm{c}} \\
2006^{\mathrm{c}} \\
2008^{\mathrm{c}} \\
2009^{\mathrm{a}} \\
2005^{\mathrm{a}}\end{array}$ \\
\hline Junho & $\begin{array}{c}(6) \\
2007^{\mathrm{a}} \\
2008^{\mathrm{a}} \\
2001^{\mathrm{b}} \\
2005^{\mathrm{b}}\end{array}$ & $\begin{array}{c}(5) \\
2007^{\mathrm{a}} \\
2008^{\mathrm{a}} \\
2001^{\mathrm{b}} \\
2005^{\mathrm{b}}\end{array}$ & $\begin{array}{c}(5) \\
2007^{\mathrm{a}} \\
2008^{\mathrm{a}} \\
2009^{\mathrm{c}} \\
2003^{\mathrm{c}} \\
2004^{\mathrm{c}} \\
\text { outros } \\
\text { anos }^{\mathrm{d}} \\
2001^{\mathrm{b}} \\
2005^{\mathrm{b}}\end{array}$ & $\begin{array}{c}(6) \\
2007^{\mathrm{a}} \\
2008^{\mathrm{a}} \\
2003^{\mathrm{b}} \\
2001^{\mathrm{c}} \\
2005^{\mathrm{d}}\end{array}$ & $\begin{array}{c}(6) \\
2007^{\mathrm{a}} \\
2008^{\mathrm{b}} \\
2003^{\mathrm{e}} \\
\text { outros } \\
\text { anos }^{\mathrm{f}} \\
2001^{\mathrm{c}} \\
2005^{\mathrm{d}}\end{array}$ & $\begin{array}{c}(7) \\
2007^{\mathrm{a}} \\
2008^{\mathrm{b}} \\
2003^{\mathrm{c}} \\
2001^{\mathrm{d}} \\
2005^{\mathrm{e}}\end{array}$ & $\begin{array}{c}(6) \\
2007^{\mathrm{a}} \\
2008^{\mathrm{a}} \\
2001^{\mathrm{b}} \\
2005^{\mathrm{c}}\end{array}$ & $\begin{array}{c}(7) \\
2008^{\mathrm{a}} \\
2007^{\mathrm{a}} \\
2000^{\mathrm{b}} \\
2001^{\mathrm{c}} \\
2005^{\mathrm{d}}\end{array}$ & $\begin{array}{c}(6) \\
2008^{\mathrm{a}} \\
2007^{\mathrm{a}} \\
2004^{\mathrm{a}} \\
2010^{\mathrm{b}}\end{array}$ \\
\hline Julho & $\begin{array}{c}(7) \\
2007^{\mathrm{a}} \\
2000^{\mathrm{b}}\end{array}$ & $\begin{array}{c}(7) \\
2007^{\mathrm{a}} \\
2000^{\mathrm{b}}\end{array}$ & $\begin{array}{c}(6) \\
2007^{\mathrm{a}} \\
2000^{\mathrm{b}} \\
2006^{\mathrm{c}}\end{array}$ & $\begin{array}{c}(6) \\
2007^{\mathrm{a}} \\
2000^{\mathrm{b}} \\
2005^{\mathrm{c}} \\
2006^{\mathrm{c}}\end{array}$ & $\begin{array}{c}(7) \\
2007^{\mathrm{a}} \\
2005^{\mathrm{b}} \\
2006^{\mathrm{b}}\end{array}$ & $\begin{array}{c}(8) \\
2007^{\mathrm{a}} \\
2005^{\mathrm{b}} \\
2006^{\mathrm{b}}\end{array}$ & $\begin{array}{c}(6) \\
2007^{\mathrm{a}} \\
2002^{\mathrm{b}} \\
2000^{\mathrm{b}}\end{array}$ & $\begin{array}{c}(7) \\
2007^{\mathrm{a}}\end{array}$ & $\begin{array}{c}(6) \\
2007^{\mathrm{a}} \\
2000^{\mathrm{b}} \\
2006^{\mathrm{c}} \\
2005^{\mathrm{c}}\end{array}$ \\
\hline
\end{tabular}


VANZ, A. et al. Estudo preliminar de características oceanográficas e...

A variação de TSM é ampla ao longo da área de estudo no período da pesca da tainha (TABELA 3).A diferença média da TSM para os três meses oscila de $2,5^{\circ}$ a $3,0^{\circ} \mathrm{C}$ sendo as maiores observadas no P5 e P6. Maio apresenta as menores diferenças mensais entre os pontos variando de $2,6^{\circ} \mathrm{C}$ nos $\mathrm{P} 1$ e $\mathrm{P} 3$ a $1,8^{\circ} \mathrm{C}$ no $\mathrm{P} 9$. A diferença vai diminuindo dos pontos da costa do RS para os de SC sugerindo uma homogeneidade maior da TSM nas águas na costa de SC. Em junho as diferenças ficaram acima de $3,5^{\circ} \mathrm{C}$ atingindo $5^{\circ} \mathrm{C}$ no $\mathrm{P} 5$. Julho a maior diferença de TSM ocorreu no $\mathrm{P} 1$ e a menor, no P8. As diferenças para esse mês ficaram entre $3,6^{\circ} \mathrm{C}$ a $4,4^{\circ} \mathrm{C}$.

TABELA 3: Valores de TSM $\left({ }^{\circ} \mathrm{C}\right)$ máximo, mínimo e diferença dos pontos analisados.

\begin{tabular}{llrrrrrrrrr}
\hline 3 mes. & & P1 & P2 & P3 & P4 & P5 & P6 & P7 & P8 & P9 \\
\hline \multirow{7}{*}{ maio } & Máx. & 17,5 & 17,7 & 18,4 & 19,5 & 20,0 & 20,9 & 21,3 & 21,8 & 22,2 \\
& Mín. & 14,8 & 15,1 & 15,7 & 16,7 & 17,0 & 17,9 & 18,4 & 19,0 & 19,7 \\
& Dif(1) & 2,7 & 2,6 & 2,7 & 2,8 & 3,0 & 3,0 & 2,9 & 2,8 & 2,5 \\
& Dif(2) & 1,0 & 0,9 & 1,0 & 1,0 & 0,9 & 0,9 & 1,0 & 0,9 & 1,0 \\
& Máx. & 20,4 & 20,5 & 21 & 21,6 & 21,9 & 22,3 & 22,6 & 22,9 & 23,3 \\
& Mín. & 17,8 & 18 & 18,4 & 19,3 & 19,6 & 20,2 & 20,6 & 21 & 21,5 \\
& Dif(1) & 2,6 & 2,5 & 2,6 & 2,3 & 2,3 & 2,1 & 2,0 & 1,9 & 1,8 \\
& Dif(2) & 1,2 & 1,2 & 1,2 & 1,5 & 1,4 & 1,6 & 1,6 & 1,9 & 1,8 \\
\hline junho & Máx. & 18,0 & 18,2 & 19,0 & 20,2 & 20,8 & 21,5 & 22,3 & 22,7 & 22,8 \\
& Mín. & 14,5 & 14,5 & 14,8 & 15,9 & 15,8 & 16,8 & 17,4 & 18,4 & 19,4 \\
& Dif(1) & 3,5 & 3,7 & 4,2 & 4,4 & 5,0 & 4,7 & 4,9 & 4,3 & 3,5 \\
& Dif(2) & 1,6 & 1,3 & 1,2 & 1,3 & 1,1 & 1,2 & 0,8 & 1,2 & 1,5 \\
\hline julho & Máx. & 15,6 & 16,0 & 16,7 & 17,8 & 18,4 & 19,3 & 19,6 & 19,8 & 20,7 \\
& Mín. & 11,3 & 11,9 & 12,9 & 13,7 & 14,2 & 15,2 & 15,8 & 16,3 & 17,0 \\
& Dif(1) & 4,4 & 4,0 & 3,8 & 4,1 & 4,2 & 4,1 & 3,7 & 3,6 & 3,7 \\
& Dif(2) & 2,5 & 2,6 & 2,6 & 2,4 & 2,4 & 2,4 & 2,3 & 2,4 & 2,5 \\
\hline
\end{tabular}

Excluindo os anos com os valores maiores e menores de TSM (FIGURA 2) para cada mês e para o período dos três meses a diferença cai sensivelmente e representa um valor mais próximo da realidade para os anos estudados (TABELA 3 Dif(2)). Foram retirados do cálculo dos 3 meses 2005 e 2007, de maio 2009, de 
VANZ, A. et al. Estudo preliminar de características oceanográficas e...

junho 2001, 2005, 2007 e 2008, de julho 2007. As maiores diferenças caem de $3^{\circ} \mathrm{C}$ para $1^{\circ} \mathrm{C}$ para o período dos 3 meses, $2,6^{\circ} \mathrm{C}$ para $1,9^{\circ} \mathrm{C}$ em maio, $5,0^{\circ} \mathrm{C}$ para $1,6^{\circ} \mathrm{C}$ em junho e $4,4^{\circ} \mathrm{C}$ para $2,6^{\circ} \mathrm{C}$ em julho.

A TSM apresentou uma variação significativa diminuindo de maio a julho. Dos meses analisados, junho apresentou as maiores variações (FIGURA 2 e TABELA 3). Essa variação é explicada pelo encontro de águas mais frias trazidas pela Corrente das Malvinas com as águas quentes da Corrente do Brasil. Esse encontro produz muitos meandros e a ampla variação espacial e temporal de TSM. Maio com TSM mais altas é resultado da maior influencia da Corrente do Brasil que com o passar do tempo, passa a ser da Corrente das Malvinas, resultando nas baixas TSM em julho (SILVEIRA, et al., 2000).

A TABELA 4 mostra a quantidade de tainha pescada pela modalidade industrial e artesanal. Os anos que a pesca total foi mais rendosa foram 2007, 2009 e 2005 e àqueles em que a quantidade capturada foi mais modesta 2001, 2002 e 2006. A FIGURA 2 mostra que 2007 apresentou TSM baixas, 2005 altas e 2009 intermediárias. TSM mais baixas favorecem a safra da tainha, podendo atingir praticamente o dobro daquelas com TSM altas (TABELA 4). O senso comum entre os pescadores é que o frio aumenta a possibilidade de safras ótimas de tainha e apesar de 2007 sugerir isso, essa relação não é tão simples. Para a modalidade artesanal, os números mostram que essa relação é inversa, ou seja, tivemos volumes maiores capturados em 2005 (TMS altas) e menores em 2007 (TSM baixas).

Segundo a imprensa, a safra de 2007 foi marcante. As condições climáticas foram favoráveis e todas vieram no momento e na intensidade certa, o que resultou numa das melhores dos últimos 20 anos, quando foram capturadas mais de 2,2 mil toneladas do peixe no litoral de Santa Catarina (WINCK, 2008). A estatística da pesca do IBAMA (TABELA 4) mostra que foi o triplo dos valores mencionado pela imprensa. Isso mostra a 
VANZ, A. et al. Estudo preliminar de características oceanográficas e...

dificuldade de se obter informações precisas sobre o volume de tainha capturado.

TABELA 4: Estatística pesca da tainha industrial e artesanal para o estado de Santa Catarina.

\begin{tabular}{cccc}
\hline ano & Industrial (t) & Artesanal (t) & total \\
\hline 2000 & $2620,6^{*}$ & - & 2620,6 \\
2001 & 947,0 & 223,0 & 1170,0 \\
2002 & 1346,0 & 239,0 & 1585,0 \\
2003 & 2480,5 & 257,0 & 2737,5 \\
2004 & 2642,0 & 275,0 & 2917,0 \\
2005 & 3115,5 & 298,0 & 3413,5 \\
2006 & 1081,0 & 198,0 & 1279,0 \\
2007 & 6396,5 & 225,0 & 6621,5 \\
2008 & $2038,3^{*}$ & - & 2038,3 \\
2009 & $4121,8^{*}$ & - & 4121,8 \\
\hline
\end{tabular}

Fonte: Disponível http://www.ibama.gov.br/* UNIVALI/CTTMar, 2010.

A TSM de 2008 foi muito próxima daquela registrada para 2007, entretanto foi apontada pelos pescadores, como sendo uma das piores da história recente (WINCK, 2008). Essa noticia não esta de acordo quanto ao volume capturado que foi de 2038,3 ton. só na modalidade industrial (TABELA 4).

Embora, a TSM baixa favoreça a pesca da tainha (Ex. 2007), isso não pode ser tomado como certo, pois anos com TSM baixa podem ter safras ruins, como 2008 e anos com TSM altas, safras boas como 2005.

Com o volume pescado pela frota industrial e os dados de TSM média mensal de cada ano foi feito gráficos de correlação. A correlação é fraca e fortemente condicionada ao valor de 6396,5 ton. capturadas em 2007. Verificou-se timidamente que com a diminuição da TSM ocorre um aumento no volume de tainha capturado com leve tendência de ficar mais forte em junho e julho. Entretanto, em maio ela é inversa, ou seja, ocorre um aumento no volume capturado com o aumento de TSM. Com a pesca artesanal as conclusões foram semelhantes. 
VANZ, A. et al. Estudo preliminar de características oceanográficas e...

PAES et. al., (2009) estudaram a relações entre as anomalias de TSM na costa sul brasileira e os desembarques de tainha no RS identificaram periodicidades interanuais da captura de tainha correlacionadas significativamente com as variações temporais das anomalias de temperatura superficial do mar. O período de mais representativo foi o anual e seguido de uma variação significativa bi-anual.

GARCIA et. al., (2003) observaram que a composição dos peixes da Lagoa dos Patos é influenciada pelos fenômenos ENOS (El Niño Oscilação Sul). Este fenômeno influencia o regime de chuvas no Rio Grande do Sul causando um aumento da vazão da Lagoa dos Patos dificultando a entrada de juvenis de diversas espécies, e possivelmente os de tainha, pois esses utilizam a Lagoa dos Patos para crescimento.

PAES et. al., (2010) estudaram as influencias do ENSO e da Oscilação Antártica (AAO - anomalias do campo de pressão atmosférica no hemisfério sul de $20^{\circ}$ a $\left.60^{\circ} \mathrm{S}-\mathrm{MO}, 2000\right)$ na variação interanual de juvenis de tainha no estuário da Lagoa dos Patos. Verificaram que $31 \%$ da variação temporal (interanual) da tainha é devido exclusivamente à $\mathrm{AAO}$ e que o El Nino explica apenas $14 \%$ e a interação entre Nino 3 e AAO é responsável por $30 \%$ dessa variação. Esses autores sugerem que o padrão encontrado pode estar revelando uma influencia positiva da $\mathrm{La}$ Nina que ocorre após o El Nino magnificada pela interação com a AAO. Na La Nina não se verifica o bloqueio atmosférico e com as passagens das frentes frias a circulação superficial na região se dá em direção à costa o que favoreceria o transporte de larvas de tainha para o interior do estuário aumentando sua abundância 2 a 3 anos após quando atingiria novamente sua maturidade reprodutiva.

Esses estudos sugerem que em anos de La Nina ocorre um aumento no recrutamento de larvas para o estuário. Além disso, o maior volume capturado em 2007 foi em um ano de La Nina forte sugerindo que em anos muito frios ocorrem melhores safras. Esses estudos apontam que fenômenos globais e não somente locais/regionais influenciam na pesca da tainha. 
VANZ, A. et al. Estudo preliminar de características oceanográficas e...

\section{Ventos}

Os dados de ventos apresentam muitas lacunas e devem ser observados com cautela. A TABELA 1 mostra que, com exceção da estação de Itajaí, as demais, não têm dados que cobrem todo o período estudado. Por isso optamos por trabalhar com valores médios mensais para cada quadrante (Norte, Leste, Sul e Oeste) para a velocidade do vento para cada estado ( $\mathrm{SC}$ e RS). No cálculo da média foi utilizados dados de vento de 5 estações meteorológicas costeiras para cada estado (FIGURA 3).
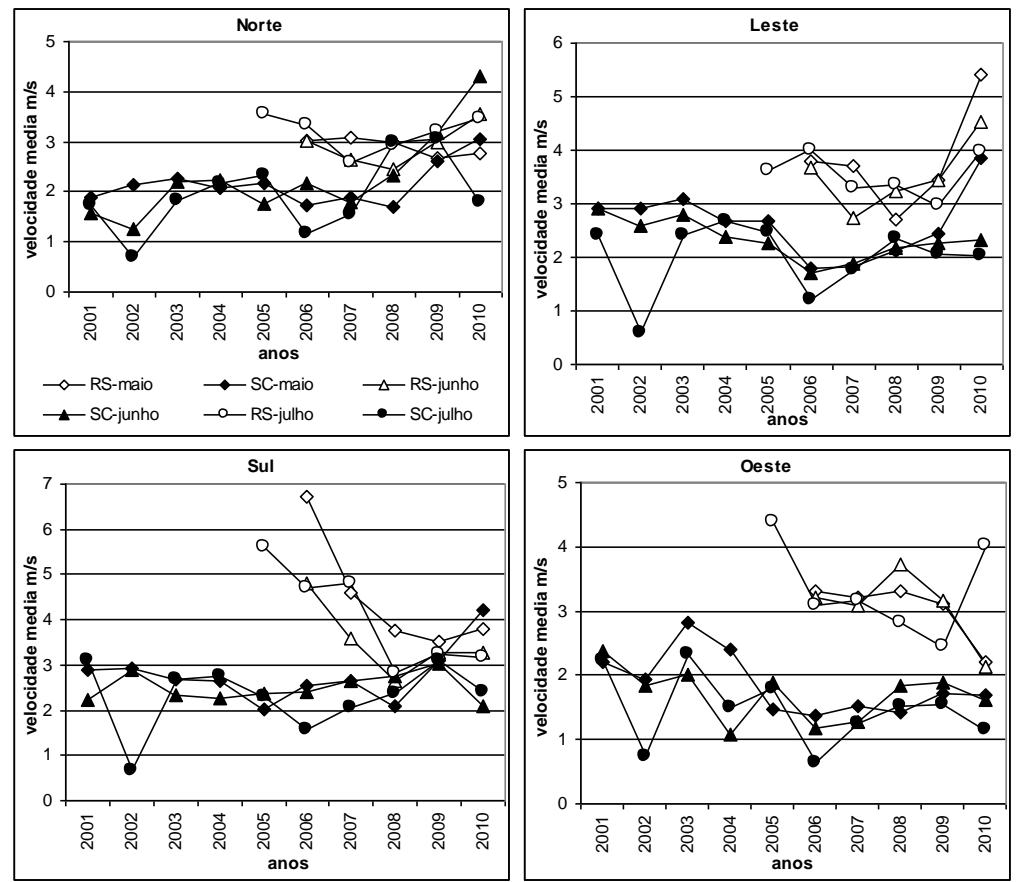

FIGURA 3: Velocidade média mensal do vento por quadrante. 
VANZ, A. et al. Estudo preliminar de características oceanográficas e...

As velocidades médias do vento para SC ficaram abaixo de $3 \mathrm{~m} / \mathrm{s}$, com predominância de valores próximos a $2 \mathrm{~m} / \mathrm{s}$, oscilando para valores maiores para o quadrante Sul e Leste e menores, para o quadrante Oeste. Para RS as velocidades são maiores, entre 3 e com a $4 \mathrm{~m} / \mathrm{s}$, chegando próximo de $7 \mathrm{~m} / \mathrm{s}$ no quadrante $S$ ul.

A Figura 4 mostra a porcentagem da média mensal dos ventos por quadrante das estações meteorológicas costeiras do
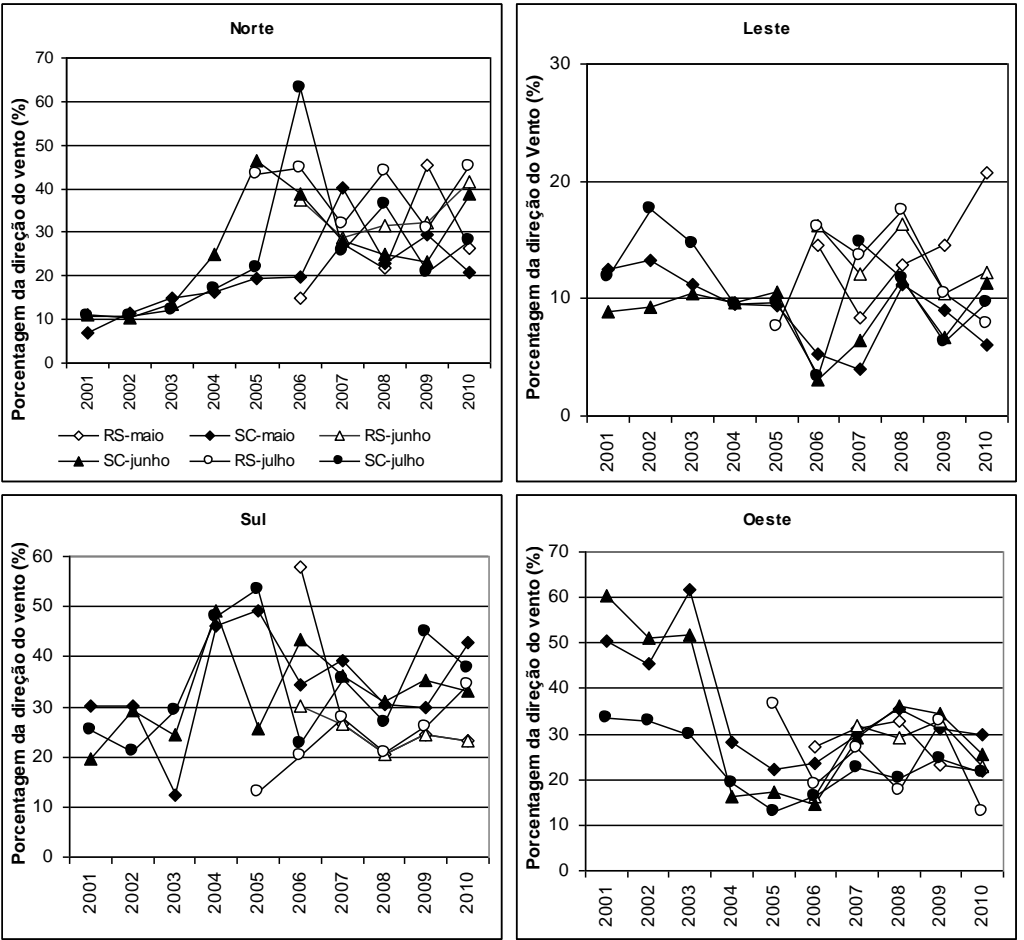

estado de SC e do RS.

FIGURA 4: Porcentagem da direção do vento por quadrante.

Em SC houve um aumento gradativo na porcentagem de vento do quadrante Norte de 2001 a 2005, depois, ficou entre 20 e 
VANZ, A. et al. Estudo preliminar de características oceanográficas e...

40\%. Em 2006 esses valores ficaram acima de 60\% para o mês de julho.

Para o quadrante Sul, para SC, os anos de 2004 e 2005 registraram as porcentagens mais altas. Antes de 2004 às porcentagens não ultrapassaram a 30. Após 2005, na maioria dos meses, as porcentagens ficaram entre 20-40 para os dois estados, com tendência de serem maiores para SC.

O quadrante Leste apresentou as menores variações de porcentagens, abaixo de 20 e o quadrante Oeste as porcentagens mensais diminuem até 2005, permanecendo em torno entre 10 e 20 nos anos subseqüentes. Chama a atenção os valores altos para os meses maio e junho nos anos 2001, 2002 e 2003 para SC.

A direção do vento variou bastante nos anos estudados. Com exceção do quadrante Leste os outros quadrantes apresentaram diferenças de até $50 \%$, por exemplo, para o quadrante Norte o mês julho em 2001 foi 10\% e em 2006, mais de 60\% (FIGURA 4).

\section{Ciclones}

No Sul do Brasil a passagem de ciclones extratropicais produz intensos ventos em superfície, nas direções sul, sudoeste ou sudeste, ocasionando agitação marinha junto a costa catarinense (CAMARGO \& HARARI, 1994). A altura e direção das ondas dependem da posição, trajetória e intensidade dos ciclones no mar. Quanto maior a intensidade e a pista de vento, maior será o efeito do vento sobre a superfície marítima e consequentemente, a ondulação (APEL, 1990). Segundo GAN \& RAO (1991), no litoral Sul do Brasil, os ciclones extratropicais são mais frequentes entre abril e setembro com média de 2 casos mensais.

A TABELA 5 mostra os dias e os meses que ciclones influenciaram as condições marítimas durante os anos de estudo. $\mathrm{O}$ número de ciclones registrado foi $9 \mathrm{em}$ maio, $5 \mathrm{em}$ junho e $2 \mathrm{em}$ julho. $\mathrm{O}$ ano que mais passou ciclones foi o de 2010, seguido de 2000 e 2009. Nos anos de 2002 e 2005 nenhum ciclone foi registrado no período da pesca da tainha. 
VANZ, A. et al. Estudo preliminar de características oceanográficas e...

TABELA 5: Número de ciclones anuais registrados no período de pesca.

\begin{tabular}{llc}
\hline Ano & \multicolumn{1}{c}{ Dias/mês } & Quant. \\
\hline 2000 & $16,17,18$ maio; 25,26 de maio e 11,12,13,14 de julho & 3 \\
2001 & $16,17,18,19$ de maio & 1 \\
2002 & & - \\
2003 & $24,25,26$ de maio & 1 \\
2004 & 15,16 de maio e 26,27 de maio & 2 \\
2005 & & - \\
2006 & 01 de junho; 27 de junho & - \\
2007 & 15,16 de junho & 1 \\
2008 & 24,25 de maio & 1 \\
2009 & 01 de junho; 30 de junho e 08 de julho & 3 \\
2010 & 20 de maio; 26 de maio; 31 de maio; 06 de junho & 4 \\
\hline
\end{tabular}

O número de ciclones no período de pesca variou de 0 a 4 (TABELA 5) e a influencia desses no volume de tainha capturado não fica evidente. Dois anos, 2005 e 2007, foram marcados pelo baixo número de ciclones, 0 e 1 respectivamente, com volume pescado significativo. Por outro lado, 2009 com 3 ciclones apresentou o segundo maior volume capturado somente na modalidade industrial. $O$ que pode explicar essa relação é a intensidade e a duração do ciclone, quando esse sistema tem intensidade e duração grandes, a pesca fica prejudicada uma vez que com o mar agitado dificulta as operações a bordo. Os ciclones geram ondas acima de $1,5 \mathrm{~m}$ impedindo que se faça o cerco na pesca artesanal influenciando o volume final capturado. Infelizmente, a falta do volume pescado em 2010 poderia fornecer outros indícios e talvez estabelecer uma correlação mais clara entre estas grandezas, uma vez que, nesse ano registramos o maior número de ciclones do período de estudo. 
VANZ, A. et al. Estudo preliminar de características oceanográficas e...

\section{Ondas}

Os ciclones e as frentes frias são os principais sistemas que produzem a agitação marítima que dificultam a pesca da tainha, principalmente, a artesanal.

Dados reais de ondas na região sul do Brasil são poucos. MELO et al., (2002) analisando os meses de maio, junho e julho encontram com frequência ondas com 1,5 e 2,0m e muitos eventos com direção dominante do quadrante $\operatorname{Sul}\left(180^{\circ}\right)$.

ARAÚJO et al., (2003) verificaram que no outono, estação que ocorre a pesca da tainha, o valor modal de Hs é de $1.5 \mathrm{~m}$ e no inverno o Hs varia de 1.25 a $2.5 \mathrm{~m}$.

STRAUCH et. al., (2009) estudaram o padrão das ondas de verão e outono no Litoral Sul e Norte do RS e verificaram que no outono em Tramandaí, os resultados indicaram alturas significativas com predominância para a direção de SSE, atingindo as maiores freqüências os valores entre 1,0 e 2,0m. Em Rio Grande as alturas significativas ocorrem no quadrante SE, atingindo valores máximos de 5,0m com um progressivo aumento de freqüência da direção E para SSE.

Recentemente, PIANCA et. al., (2010) apontam que para a Região Sul a direção dominante da onda é de $S$, com alturas entre 2 - 3m e períodos de 10-12 s.

\section{Conclusão}

O trabalho apresentou um panorama geral de algumas características oceanográficas e meteorológicas que atuam no período da pesca da tainha. Em anos mais frios (La Nina forte) a pesca foi mais significativa. A diminuição continua da TSM de maio a julho favorece boas safras (Ex. 2007), entretanto anos de TSM quentes (Ex. 2005) também podem ter safras expressivas.

A predominância mensal do vento pode ser de até $60 \%$ para os quadrantes norte, sul e oeste e de $20 \%$ para o leste.

$\mathrm{O}$ número de ciclones que passa durante o período de pesca não afeta significativamente o volume final pescado porque sua 
VANZ, A. et al. Estudo preliminar de características oceanográficas e...

frequência é maior em maio e, segundo os pescadores, a pesca é mais abundante em junho e julho.

No período de pesca da tainha é comum ondas com 1,5 a 2,0m de altura.

Há uma alternância entre safras boas e ruins evidente nos últimos anos, especialmente para a pesca industrial.

\section{Referências bibliográficas}

APEL, J.R. Principles of Ocean Physics. 3. ed, Academic Press, Inc, London, 1990. 631p.

ARAÚJO, C.E.; FRANCO, D.; MELO FO, E. \& PIMENTA, F.M.; Wave regime characteristics of the Southern Brazilian Coast. In: Proceedings of the Sixth International Conference on Coastal and Port Engineering in Developing Countries, COPEDEC VI, Colombo, Sri Lanka, 2003, p. 15.

CAMARGO, R.; HARARI, J. 1994. Modelagem Numérica de Ressacas na Plataforma Sudeste do Brasil a partir de Cartas Sinóticas de Pressão Atmosférica na Superfície. Boletim do Instituto Oceanográfico, 42(1), 19-34.

GAN, M.A.; RAO, V.B. Surface cyclogenesis over South America. Monthly Weather Review, v.119, n.5, p.1293-1302, 1991.

GARCIA, A.M.; VIEIRA, J.P. \& WINEMILLER, K.O. 2003. Effects of 1997-1998 El Niño on the dynamics of the shallowwater fish assemblage of the Patos Lagoon estuary (Brazil). Estuarine, Coastal and Shelf Science. 57: 489-500.

IBAMA 2007 - Estatística da pesca em 2007. Brasil - Grandes regiões e unidades da federação. Brasília-DF, 2007. em: http://www.ibama.gov.br/ Acesso em: 02 set. 2007. 
VANZ, A. et al. Estudo preliminar de características oceanográficas e...

IBAMA/ICMBIO/CEPSUL 2007 - Relatório de Reunião Técnica para o Ordenamento da Pesca de Tainha (Mugil platanus, $M$. liza) na Região Sudeste/Sul do Brasil, Itajaí, 2007.

MELO FO, E., J.H.G.M. ALVES, R.C. BARLETTA, F.V. BRANCO, D. FRANCO, G. R. HAMMES, F.M. PIMENTA, D.A.R. MENDES, E. PRIDO, C.E.A. SALLES, A.C. SOUTO. Implementação do "Programa de Informação Costeira - on line". Workshop Diretrizes Técnicas para Modelagem do Derramamento de Óleo no Mar, IBAMA/ANP, Rio de Janeiro, pp 24, 2002 (Publicado em CD, sem paginação).

MENEZES, N. A. \& FIGUEIREDO, J. L. 1985. Manual de peixes marinhos do sudeste do Brasil. V. Teleostei. 4: 105 p. Museu de Zoologia da Universidade de São Paulo, São Paulo, SP.

MO, K.C., 2000: Relationships between Low-Frequency Variability in the Southern Hemisphere and Sea Surface Temperature Anomalies. Journal of Climate, 13, 3599-3610.

OCCHIALINI, D. - Informações técnico-científicas apresentadas para subsidiar a proposta de ordenamento da Pesca de Tainha no Litoral Sudeste e Sul do Brasil. In: Relatório de Reunião Técnica para o Ordenamento da Pesca de Tainha (Mugil platanus, M. liza) na Região Sudeste/Sul do Brasil, Itajaí, 2007.

PAES, E.T.; PERES, M. B.; GHERARDI, D.F. M \& SOUZA, R.B. Relações entre as anomalias de temperatura superficial do mar na costa sul brasileira e os desembarques de tainha (Mugil Platanus GÜNTHER, 1880). Anais XIV Simpósio Brasileiro de Sensoriamento Remoto, Natal, Brasil, 2009, INPE, p. 66276632 .

PAES, E.T.; VIEIRA, J.P. \& GARCIA, A.M. Influências do Enso e Oscilação Antártica na Variação Interanual de Juvenis de Tainha 
VANZ, A. et al. Estudo preliminar de características oceanográficas e...

(Mugil Platanus Günther, 1880) no Estuário da Lagoa dos Patos. III Congresso Brasileiro de Oceanografia - CBO'2010. Rio Grande (RS), 2010, p. 1222-1225.

PIANCA, C.; MAZZINI, P.L.F. \& SIEGLE, E., 2010. Brazilian Offshore Wave Climate Based On Nww3 Reanalysis. Brazilian Journal Of Oceanography, 58(1):53-70.

SILVEIRA, I.C.A.; SCHMIDT, A.C.K.; CAMPOS, E.J.D. GODOI, S. S. \& IKEDA, Y. 2000. A Corrente do Brasil ao Largo da Costa Leste Brasileira. Revista Brasileira de Oceanografia. 48(2):171-183.

STRAUCH，J.C.; CUCHIARA， D.C.; TOLDO JR, E.E. \& ALMEIDA, L.E.S.B. (2009). O Padrão das Ondas de Verão e Outono no Litoral Sul e Norte do Rio Grande do Sul. Revista Brasileira de Recursos Hídricos. 14(4), 29-37.

UNIVALI/CTTMar, 2010. Boletim estatístico da pesca industrial de Santa Catarina - Ano 2009 e panorama 2000 2009. Universidade do Vale do Itajaí, Centro de Ciências Tecnológicas da Terra e do Mar, Itajaí, SC. 97 p.

VIEIRA, J.P. \& SCALABRIN, C. 1991. Migração reprodutiva da "tainha" (Mugil platanus Günther,1880) no sul do Brasil. Atlântica. 13 (1): 131-141.

VIEIRA, J.P. 1985. Distribuição, abundância e alimentação dos jovens de Mugilidae no estuário da Lagoa dos Patos e movimentos reprodutivos da "tainha" (Mugil platanus, Günther, 1880) no litoral sul do Brasil.. Dissertação (Mestrado em Oceanografia Biológica) - Fundação Universidade do Rio Grande. RS. 104p.

VIEIRA, J.P.; GARCIA, A.M. \& GRIMM, A.M. (no prelo). Preliminary evidences of El Niño effects on the mullet fishery of 
VANZ, A. et al. Estudo preliminar de características oceanográficas e...

Patos Lagoon estuary (Brazil). Brazilian Archives of Biology and Technology.

WINCK, A. 2008. Jornal Folha do Norte da Ilha. http://www.fundacentro.sc.gov.br/acquaforum/principal/ver_notici as.php?not=2387.

Recebido em outubro de 2011 Aceito em abril de 2012 